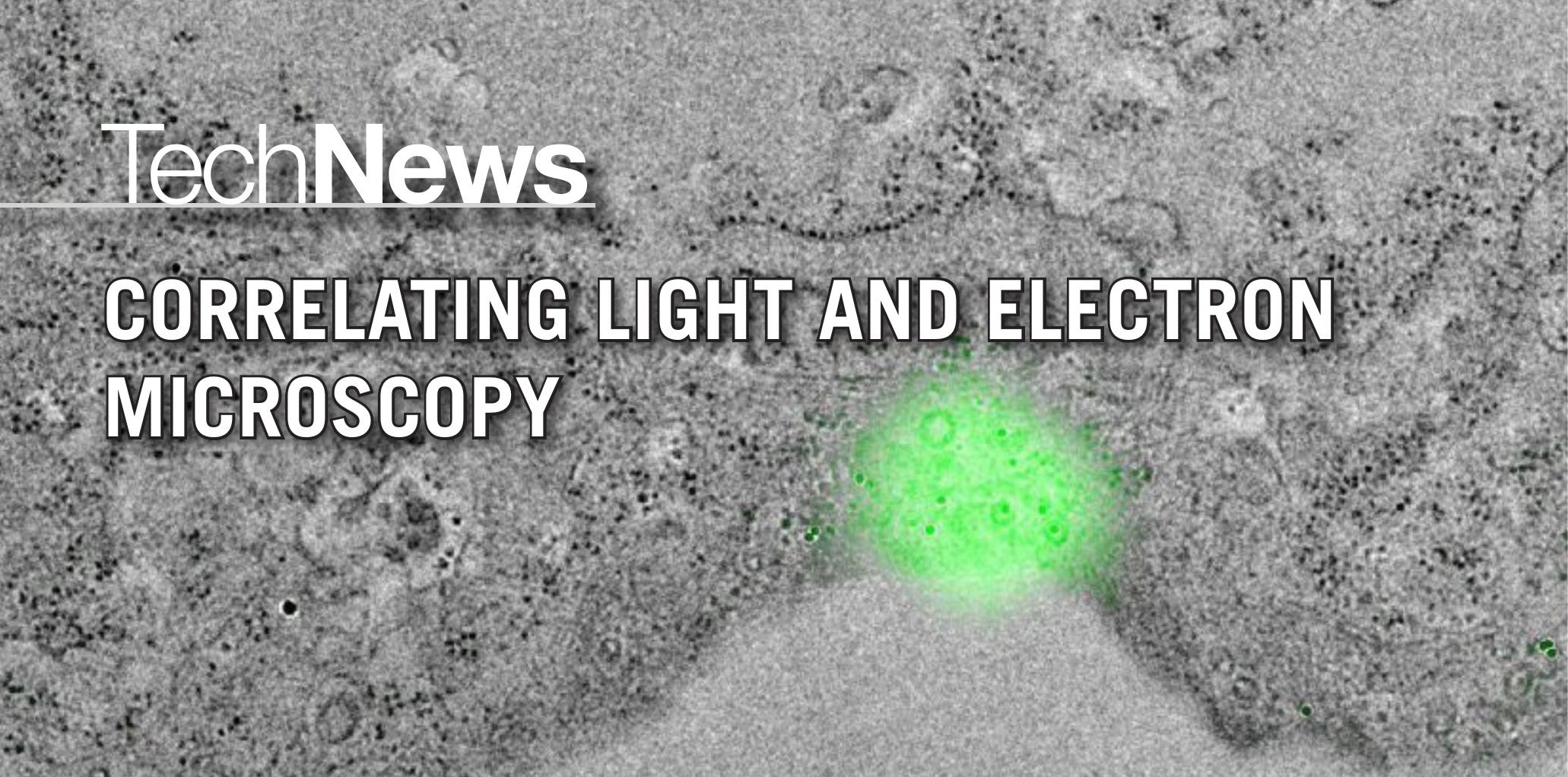

Correlating the molecular specificity of light microscopy with the high structural resolution of electron microscopy can be a challenge, Jeffrey Perkel looks at the ways researchers are bringing togetherr these methods - and the wondrous cellular details being revealed.

HUVEC endothelial cell sections stained for caveolin, a protein that assembles around caveoli (100-nm endocytic vesicles), visualized via

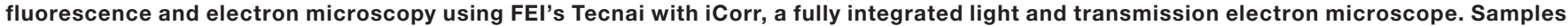
courtesy of Elly van Donselaar and Matthia Karreman, Utrecht University, the Netherlands. Image courtesy of FEl.

W en Eric Betzig and colleagues first described their new microscopy method, PALM, they chose to highlight its power by comparing it to an ultra-high-resolution approach: transmission electron microscopy (TEM).

PALM, or photoactivated localization microscopy, is a super-resolution fluorescence technique allowing users to circumvent the $200 \mathrm{~nm}$ diffraction limit that constrains optical microscopy, mapping fluorophores to within $10 \mathrm{~nm}$ or so. It produces exceptionally sharp images of the distribution of specific molecules in a cell. The only problem is that the resulting signals are nothing more than bright dots on a black background-"little blobs floating in darkness," as Harald Hess, a colleague of Betzig at Howard Hughes Medical Institute's Janelia Farm Research Campus and co-author with Betzig on the first PALM article, describes it.

Hess, Betzig and their colleagues were stuck with a question: where exactly in the cell were those blobs located? Since they were imaging fluorescent proteins coupled to cytochrome c oxidase, the team anticipated a mitochondrial distribution. But mitochondria weren't visible in the images -in fact, none of the cellular architecture was. So, Hess and his colleagues worked out a way to image their sample using both PALM and TEM, superimposing the red fluorescent staining on a grayscale TEM canvas.
It wasn't easy. Samples typically are subjected to light or electron microscopy (EM), but rarely both. The methods require different sample preparation, fixation, and environmental conditions, and a TEM sample will rarely fluoresce. Even if they could work out experimental conditions to satisfy both methods, researchers still needed to merge the resulting data sets-that is, to find the same field of view so the images can be overlaid.

"It was a heroic effort," Hess recalls, but it worked out.

Today, that kind of effort-generically called "correlative light and electron microscopy" (CLEM) - is easier. And researchers and developers, ever cognizant of the value of applying orthogonal approaches to a single problem, have now devised new tools to simplify and enhance the workflow.

\section{CLEM: A Primer}

CLEM is nothing more than the application of both light and electron microscopy to the same sample. The results can provide the best of both worlds, Hess says, blending the molecular specificity of fluorescence microscopy with the broad ultra-structural detail obtained with EM. "They really complement each other as best as you possibly could imagine." 
Kirk Czymmek, Director of North American Applications and Labs at Carl Zeiss Microscopy, says CLEM offers three specific benefits: It provides different views of the same sample, facilitates analysis of rare structures or cells, and reveals structural context. Researchers can use the approach to, for instance, determine whether a particular membrane-associated protein lies inside or outside a cell or organelle; select specific fluorescently stained cells for detailed structural interrogation; and probe the molecular stoichiometry and heterogeneity of subcellular features.

In principle, CLEM doesn't sound too difficult: Obtain a relatively low-resolution image under a light microscope, then transfer the sample to an electron microscope for an extreme cellular close-up-think of it as zooming from country level to street view in Google Maps.

In practice, however, the process is far more complicated. For one thing, says Czymmek, researchers typically are trained in one branch of microscopy or the other, meaning cooperation or collaboration between laboratories may be required.

The more pressing problems, though, are technical. First, while there are exceptions, the gentle sample-preparation conditions that work for light microscopy are insufficient for electron microscopy. Conversely, samples suitable for EM often are no longer amenable to optical imaging, as the heavy metals and chemicals used to fix and stain EM samples in many cases quench fluorescence. Plus, the processing that generally must take place prior to EM (and after light microscopy) can subtly alter a sample's architecture and layout, making it difficult to align images. The "ugly truth," says Hess, is that "the sample preparation is really the key to everything."

EM sections are generally some 2-3 orders of magnitude thinner than light microscopy sections. The field of view is also tighter: EMs provide Angstrom-level resolution, whereas traditional light microscopy resolution is perhaps $200 \mathrm{~nm}$. And then there are the physical issueslight and electron microscopes often aren't even located in the same room. As a result, it's rarely simple to locate one particular cell that you see under fluorescence and then focus in on it in the EM.

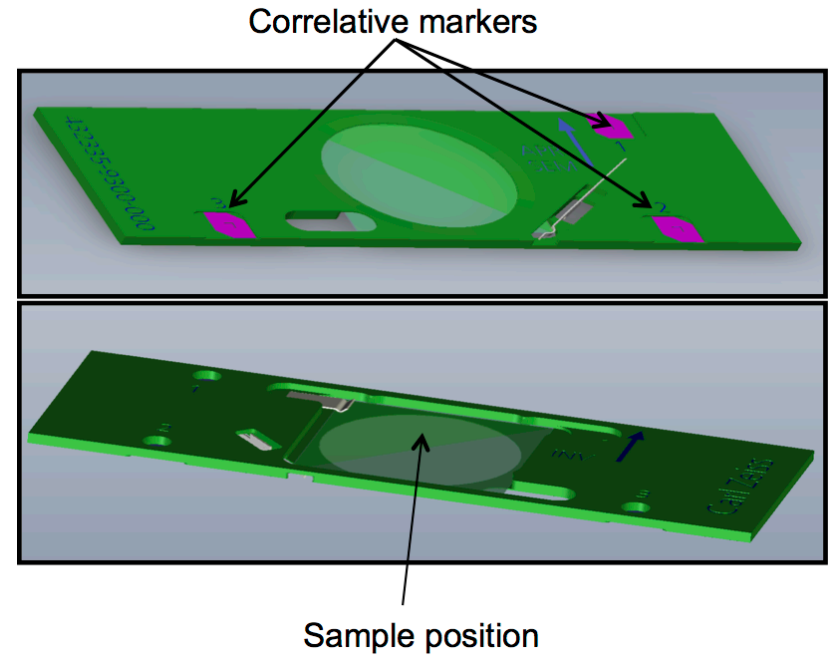

With built-in fiducials, Zeiss's Shuttle \& Find sample holder provides one solution to CLEM's so-called "retracing" problem. Photo courtesy of Carl Zeiss Microscopy.

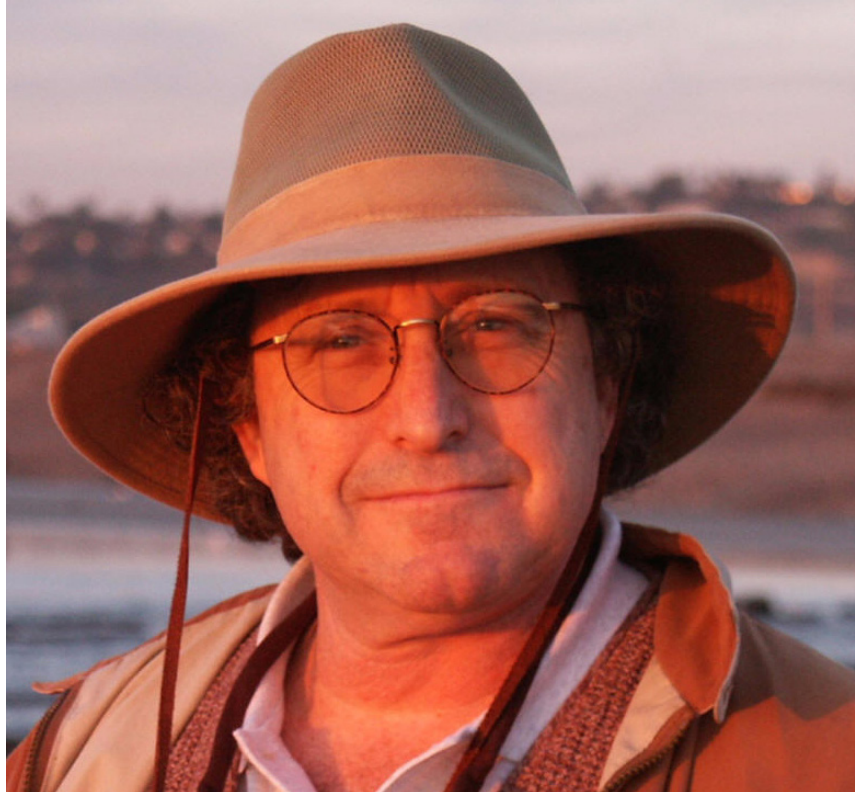

MiniSOG co-developer Mark Ellisman. Photo courtesy of Mark Ellisman.

that almost all EM labs are at least considering to do in the very near future."

The technique has sufficient interest that Paul Verkade, Reader in Cell Imaging at the University of Bristol, UK, has organized a course on the topic for the past five years. Open to 16 individuals and overseen by EMBO, the week-long course rotates students through different approaches to correlative microscopy. The biggest difficulty these students face, Verkade says, is "retracing," relocating a region of interest in one method after it was imaged in the other.

"When you are talking about carrying [a sample] between two microscopes, the biggest problem is how to find the region of interest again. There's a thousand-fold difference [in the field of view]," agrees Nalan Liv, a postdoctoral fellow at the University Medical Center in Utrecht.

One approach Verkade recommends, at least when fixing samples chemically, is using MatTek imaging dishes, which contain "an embossed finder pattern" that is visible under both light and electron illumination. These physical
alled "fiducials," and can range landmarks are sometimes called "fiducials," and can range
from a simple scratch physically etched into a coverslip or sample, to static gold or other heavy metal nanoparticles-anything visible via both imaging modalities. Carl Zeiss Microscopy's "Shuttle \& Find" system of consumables and software uses fiducial-marked sample holders manufacturer. "Over the last three years, it has moved from something that very few labs in the world did, to something
For decades, CLEM has been a niche technology. However, in the past few years the approach has gained more traction, according to Matthias Langhorst, Director of Product Marketing for Cell and Tissue Biology at FEI, an EM 
and in-development cover slips to record the positions of regions of interest during light microscopy, which can then be retrieved and revisited as the sample is moved from a light microscope to a scanning electron microscope, Czymmek says.

EM vendor FEl has commercialized an alternative strategy with its iCorr and CorrSight systems. iCorr is a TEM outfitted with low-power fluorescence microscopy optics. Users can view the sample under fluorescent illumination, identify a cell of interest, then rotate the sample holder into the EM beam path and image it at higher magnification. "Overlay is immediate and very straightforward," Langhorst says.

CorrSight, on the other hand, is "a light-microscopy platform that is especially adapted to the special sample requirements that you have in correlative microscopy." The system includes a "full-blown high-end microscope," coupled with a variety of automation tools to smooth the sample preparation process for scanning electron microscopy (SEM). One workflow allows multiple users to scan a bunch of thin sections via fluorescence to identify the few that contain a structure of interest, marking them for EM analysis. Another allows for live-cell imaging followed by chemical fixation, and a third for cryopreservation for cryo-electron microscopy. "The beauty is that the sample holder or CorrSight is compatible with the SEM stage," Langhorst says. "And we also know exactly the coordinates where the cell of interest is, so the SEM system can start with that area."

\section{Of Flavins and Reactive Oxygen}

In 2011, Xiaokun Shu in Roger Tsien's laboratory, working with Mark Ellisman and his group at the University of California, San Diego, described yet another approach to overcome the CLEM "retracing" problem.

MiniSOG, or "mini Single Oxygen Generator," is a genetically encoded fluorescent protein based not on green fluorescent protein (GFP) but the more compact LOV (light, oxygen, and voltage) domain of the phototropin photoreceptor protein. When bound to flavin mononucleotide and excited at the appropriate wavelength, miniSOG both fluoresces and produces reactive oxygen species (ROS).

It's the ROS that make miniSOG special. When diaminobenzidine (DAB) is added to the fixed cells or tissues, it reacts with the ROS, causing the DAB to polymerize at the site of miniSOG protein localization. That polymerized material is "osmiophilic"-it stains strongly with osmium tetroxide, depositing the electrondense metal and marking the site for electron

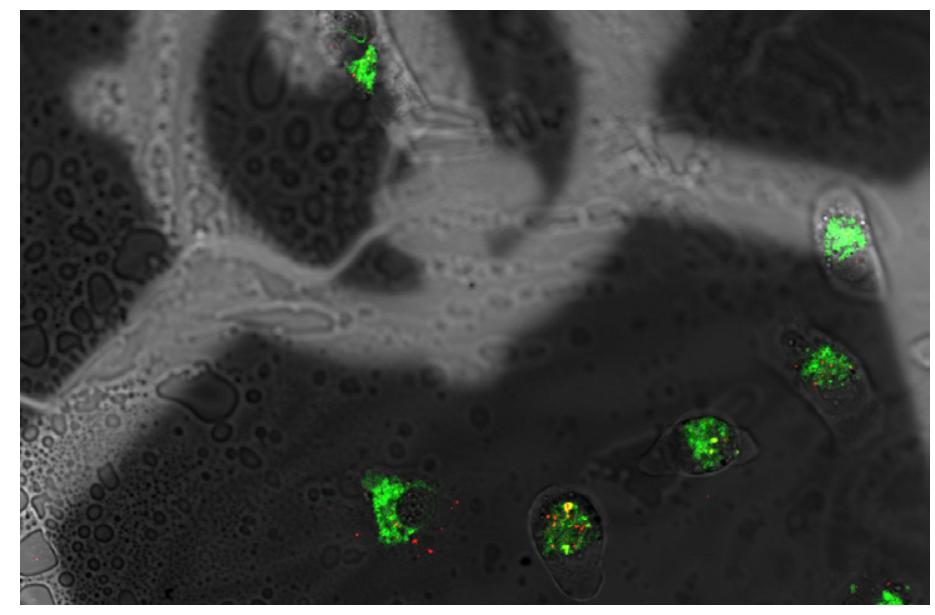

Example of a carbon-coated finder pattern to aid in the retracing of objects of interest. Photo courtesy of Paul Verkade. microscopy. The overall effect is that the cellular regions that were fluorescent under the light microscope are now also visible in the EM.

The process is akin to dusting for fingerprints, Ellisman explains. "What we're doing is dusting with metal as part of a chemical reaction, and we put a very, very thin shell of this heavy metal around the same place that was generating reactive oxygen species."

In the 2011 PLoS Biology article (1) describing the new reporter, Shu, Tsien, and Ellisman concluded, "MiniSOG may do for EM what Green Fluorescent Protein did for fluorescence microscopy." And in a sense, that has come to pass: MiniSOG has been fused to "over 100 molecules at this point" to study everything from neural connectivity in the brain to molecular trafficking, Ellisman says. The only limitation, he notes, is that while GFP is "pretty fool-proof," miniSOG requires more advanced technical expertise. "There aren't that many people around who know how to carry out high-quality sample preparation for EM anymore."

\section{Freeze Frame}

Clearly, there's no one particular way to do a CLEM study. Indeed, Verkade says one of the concepts he emphasizes to students during his CLEM course is the importance of matching method to experiment. "Think about what application you want to get out of these experiments."

Fixation, for instance, is not always the same; some users chose chemical fixation, and others opt for cryofixation. Verkade says he uses both in his own research. The former is fast, well-established, and inexpensive, but also prone to sample distortion and artifacts. Cryopreservation is cumbersome and complicated, but provides exquisite preservation of sample architecture. A third option, called Tokuyasu sectioning, blends the two, providing a way to immunostain samples following fixation.

Cryofixation involves cooling samples fast enough to prevent the formation of ice crystals, which will destroy a sample. How fast? "Several ten-thousand degrees to up to a million degrees [of cooling] per second," says Thomas Burg of the Max Planck Institute for Biophysical Chemistry in Göttingen, Germany. At that rate, water doesn't crystallize, but rather becomes "vitreous," adopting an "amorphous, glass-like" state.

Traditionally, there are two ways to achieve that state. One is to plunge a sample into a cryogen bath; the other is to freeze at high pressure. Neither works while a sample is being visualized under a light microscope.

Verkade, working with Leica Microsystems, helped develop a wheeled 


\section{Quick-RNA $^{\text {TM }}$ Kits}

\section{Purify small, large, or total RNA... You decide!}

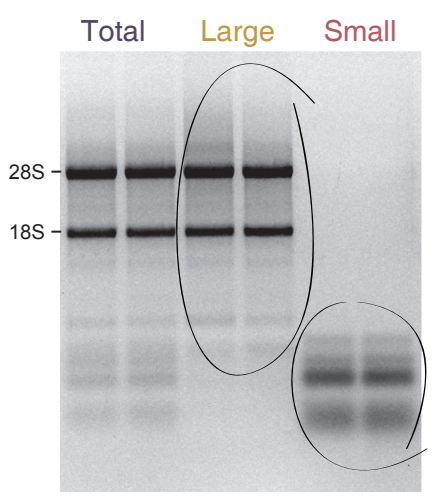

\section{DNase I included}

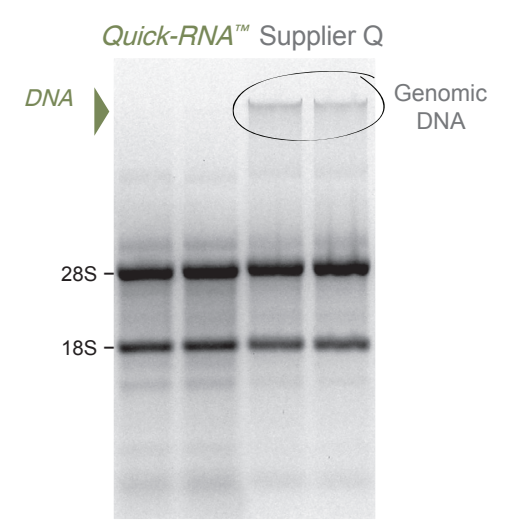

\section{RNA is ideal for RT-PCR, RNA-Seq, arrays, etc.}

\section{For Special Offers Visit}

www.zymoresearch.com

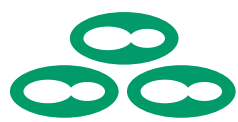

ZYMO RESEARCH

The Beauty of Science is to Make Things Simple "rapid loader" (the EMPACT2+ Rapid Transfer System) that allows him to freeze samples at high pressure within about four seconds - a time frame that enables optical sample imaging and capture of dynamic processes more or less as they happen.

Burg and coworker Yara Mejia developed an alternative approach that allows uninterrupted imaging by maintaining "an artificial non-equilibrium" between heat and cold. The system uses a microfluidic chip in which cells in a narrow channel sit atop a microfabricated electric heater, which is itself atop a liquid nitrogen-cooled silicon base. When the heater is on, the channel is warm enough for live-cell imaging; when turned off, the sample cools rapidly, losing about 10,000 degrees per second. As a result, researchers can observe their cells under optical microscopy, wait for that opportune moment, and flip a switch to cause essentially instantaneous vitrification and fixation. Burg's team was able to image yeast cells and fluorescent beads as they flowed through the channel, capturing frames every 10 milliseconds. When the heater was on, the beads looked like long-exposure astrophotography star trails. Within 10 milliseconds of turning off the heater, though, the beads froze in place, producing a sharp pattern more like the night sky.

"That is a bridge between the microscopy at room temperature for living samples, down to the low temperature when the same sample is stabilized," he says.

But the team has yet to apply the approach to CLEM itself. The sticking point, Burg says, is the "interface" between their fixation approach and the rest of the sample preparation workflow-an interface that has already been worked out for traditional freezing methods but which they have to develop for their approach. "There are a lot of intricate engineering tasks that need to be done really, really well in order to do this successfully."

\section{Superresolution Man}

Correlative microscopy isn't only about traditional light microscopy. Ellisman, for instance, blends electron microscopy with X-ray microscopy to identify regions of

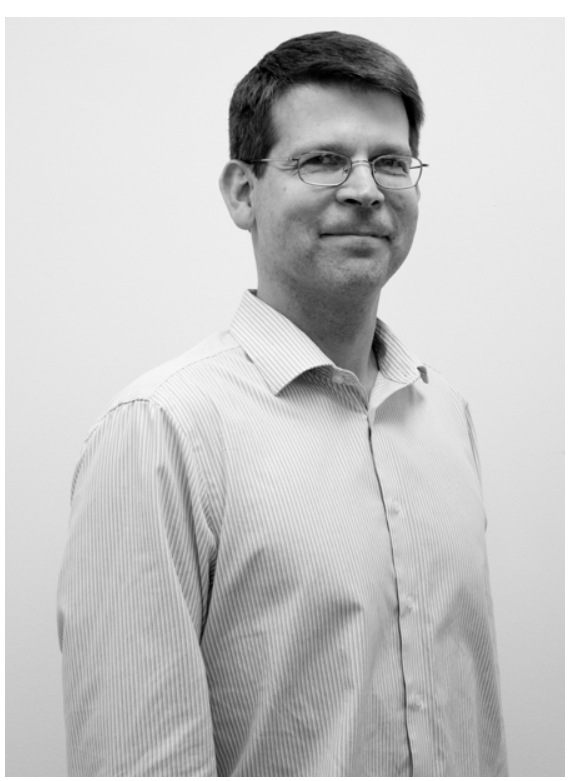

Zeiss CLEM expert Kirk Czymmek. Photo courtesy of Chelsea McCulley, Carl Zeiss Microscopy, LLC.

interest in a tissue block prior to sectioning and EM, as light cannot penetrate a tissue once its been embedded in epoxy. "We use the X-ray microscope to give us our MapQuest," he explains. "And then we move in to the right house or room in a house in this city of cells with the EM. So it's a pinpointing method."

Irit Sagi, Professor and Dean of the Graduate School at the Weizmann Institute of Science in Israel, has developed sample preparation strategies and workflows to couple B-nano's airSEM microscope with a range of orthogonal techniques, from confocal microscopy to two-photon imaging to Raman spectroscopy, and is working to integrate the technique with laser microdissection to enable 'omics-level analyses of specific cells, she says.

A methods developer himself, Hess has spent years devising ways to couple EM with super-resolution imaging. In 2012, his team coupled a 3-D interferometric PALM with focused ion beam-SEM, which uses an ion beam like a "sandblaster," Hess explains, to whittle a sample down as it is imaged, producing a 3-D EM data set. Using that approach, his team investigated the positioning of mitochondrial DNA (nucleoids) relative to the internal membranes within mitochondria themselves, finding that the nucleoids tended to reside in "little hollowed out regions where there are no cristae." 


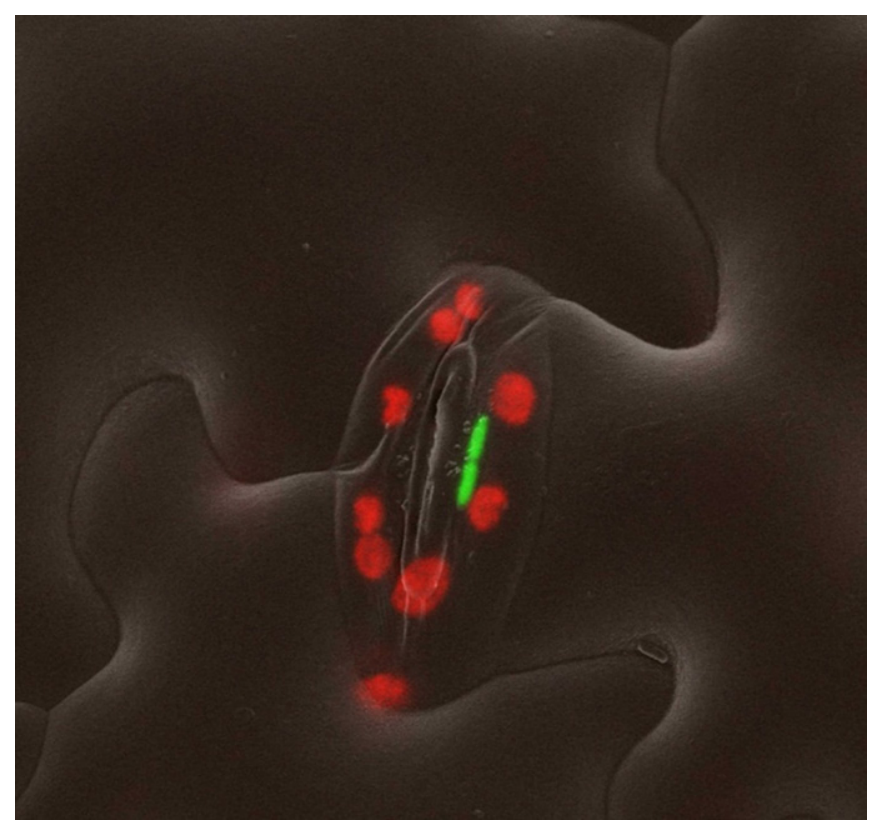

Correlative confocal/cryo-scanning electron microscopy illustrating blocked entry of the bacterial pathogen PstDC3000 (green) through stomata of Arabidopsis thaliana leaves (greyscale), Source: Kumar et al., Plant J., 72(4):694-706, 2012.

That method, though, relied on difficult to obtain instrumentation-few labs have iPALMs, Hess notes, and FIB-SEMs cost upwards of a million dollars. More recently, his lab published a "more accessible and more broadly usable" approach combining 2-D PALM with standard SEM, which he used to investigate the nuclear lamina, among other structures. A 2014 study described a way to integrate PALM with TEM that involves making a platinum replica of the cell membrane, which Hess' team then imaged at different tilt angles to create 3-D tomographic representations of clathrin-coated vesicles.

And he and others continue to innovate. Janelia Farm colleague Loren Looger, whom Hess calls "Dr. Protein Engineer," has worked with Hess to engineer a photoactivatable fluorescent protein suitable for PALM that is up to a thousand times more resistant to the EM fixative osmium tetroxide- "the most toxic, poisonous thing you can imagine to proteins," Hess says - than traditional proteins.

Not everyone will need such tools. But if you have a biological problem that could be addressed using a blend of molecular and ultra-high-resolution structural detail, it's worth a look, says Hess. "Biology is hard. You need multiple things in the tool-chest, ... different labels, different ways of looking at it."

\section{Reference}

1. Shu X., V. Lev-Ram, T.J. Deerinck, Y. Qi, E.B. Ramko, M.W. Davidson, Y. Jin, M.H. Ellisman, R.Y. Tsien. 2011. A genetically encoded tag for correlated light and electron microscopy of intact cells, tissues, and organisms. PLoS Biol. 9:e1001041.

Written by Jeffrey M. Perkel, Ph.D. IGGA

BioTechniques 57:172-177 (October 2014) doi 10.2144/000114213

\section{LIGHT SOURCES GROUP}

\section{$\Pi$ ח月L $\rightarrow$ GIOT} Commitment Like No Other

\section{Fast.}

\section{High speed modulation}

The new 56-CRN, 57-CFS, and 57-CFP series diode laser systems from Melles Griot provide excellent beam performance and pointing stability from a compact, self-contained package. This industry standard $70 \mathrm{~mm} \times 40 \mathrm{~mm} \times 40 \mathrm{~mm}$ package is a must for your OEM application by incorporating analog and digital drive electronics to provide a smaller footprint at a reduced cost.

Find out more at www.mellesgriot.com or call 1800 MG LASER
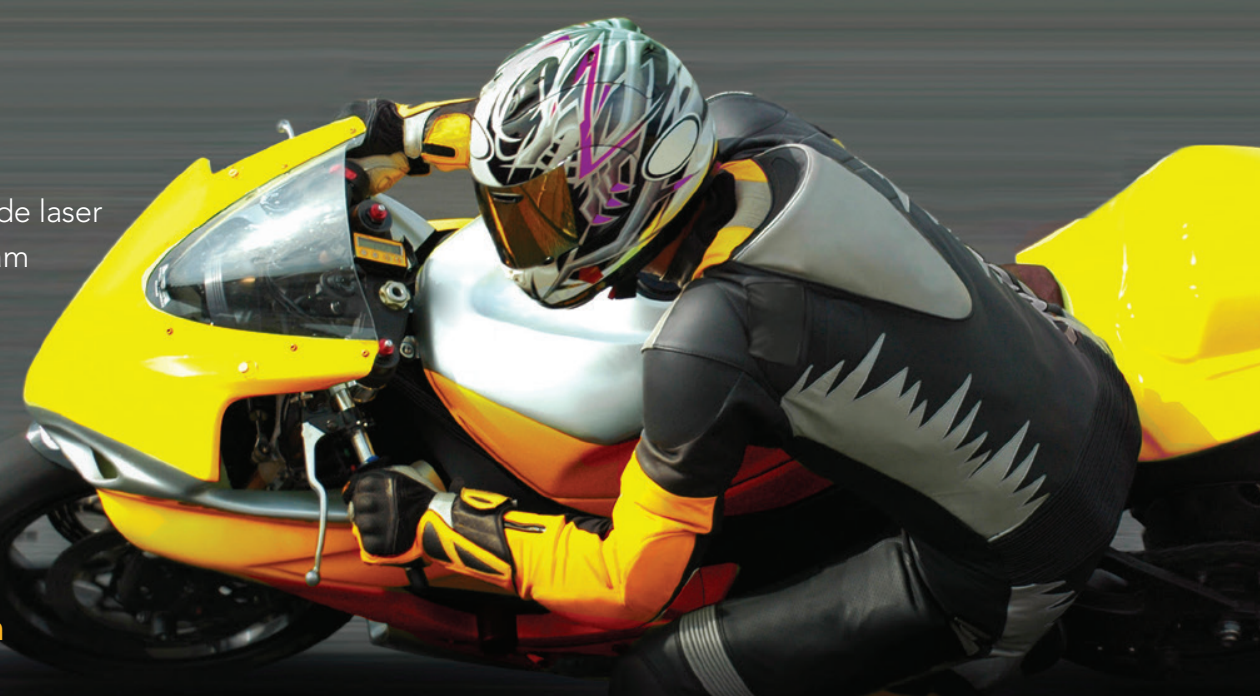